\title{
J-Curve Phenomenon-Current Understanding and Clinical Implications
}

\author{
T Govindan Unni
}

\begin{abstract}
$\mathrm{J}$-curve can be defined as an increase in event rates when the blood pressure (BP) goes below a particular level. Now that we have safe and powerful drugs available for treatment of hypertension, it has become possible to bring down the BP to very low levels. However, the concept of "lower is better" is now being questioned. Trials looking at J-curve have given conflicting results. Probably, there is no J-curve for systolic BP. J-curve for stroke and renal end points is also debatable. It is in patients with significant obstructive coronary artery disease that there are data for a J curve for diastolic BP. In such patients, we should gradually titrate the dose of drugs, carefully watching for increasing angina. Isolated systolic hypertension (ISH) is another situation wherein care has to be taken when aggressively reducing systolic BP. Even here, there are questions to be answered. The low diastolic BP could be a marker of increased aortic stiffness. Or, the low diastolic BP may be due to other associated comorbid conditions. The fear of $\mathrm{J}$-curve should not lead to undertreatment and thus deny patients the benefit of BP reduction.
\end{abstract}

Keywords: Curve, Hypertension, Hypoperfusion.

How to cite this article: Unni TG. J-Curve PhenomenonCurrent Understanding and Clinical Implications. Hypertens J 2016;2(1):16-20.

Source of support: Nil

\section{Conflict of interest: None}

From the days when primary or idiopathic hypertension was considered "essential" (because it was essential to maintain normal perfusion in vital organs and so tampering with the blood pressure may be harmful), we have come a long way. Statements like "For aught we know, the hypertension might be a compensatory mechanism that should not be tampered with even were it certain that we could control it"1 and "May not the elevation of blood pressure be a natural response to guarantee a more normal circulation to the heart, brain and kidneys" ${ }^{\prime 2}$ were famous and often quoted. The well recognized increased target organ damage secondary to abrupt reduction in blood pressure (BP) in a hypertensive emergency cautioned physicians against lowering BP to very low levels even in

\section{Professor}

Department of Cardiology, Jubilee Mission Medical College and Research Institute, Thrissur, Kerala, India

Corresponding Author: T Govindan Unni, Professor Department of Cardiology, Jubilee Mission Medical College and Research Institute, Thrissur 680005, Kerala, India, Phone: 09847308310, e-mail: unni.govindan@gmail.com a stable disease. Subsequently, the pendulum swung to the other extreme and the mantra was "lower the better." From undertreatment have we gone to overtreatment?

The J-curve phenomenon is defined as a nonlinear, quadratic increased cardiovascular disease (CVD) (ischemic heart disease, heart failure, and stroke) risk at the lower range of BP. Three reasons may explain the J-curve for diastolic BP (DBP). First, in elderly hypertensive with isolated systolic hypertension (ISH) and low DBP, wide pulse pressure (PP) itself is a risk factor. Wide PP suggests increased vessel wall stiffness and thus is a marker of increased risk. Second, the low DBP may be due to the underlying chronic debilitating diseases, which is the cause of increased risk (reverse causality). In this situation, unlike the former, there will be a narrow PP and both systolic and diastolic pressure is low. Third, in patients with significant coronary artery disease (CAD), the low diastolic pressure reduces the perfusion pressure in coronaries distal to obstruction and thus leads to ischemia. ${ }^{3}$ In the Framingham heart study, when the four components, systolic BP (SBP), DBP, mean arterial pressure (MAP), and PP were studied, only DBP showed a J-curved relationship of increased CVD risk. However, these were patients not on antihypertensive therapy and free of CVD. ${ }^{4}$

\section{BLOOD PRESSURE OR BLOOD FLOW?}

We should not confuse between "blood pressure" and "blood flow". "Hypotension" should not be confused with "hypoperfusion". Blood pressure is easy to measure, but blood flow is not. After discussing about BP for so long, we tend to forget that it is the blood flow and not $\mathrm{BP}$, which is important. The pressure inside a vessel is the product of flow and resistance. Thus, we can have low BP because of either low flow or low peripheral resistance. If it is due to low resistance, and flow is maintained, we do not have to worry about it. In fact, it may be good for the heart, as myocardial oxygen demand is reduced because of the low afterload. But, if it is the flow that is reduced, we may have problems. It was believed that the high $\mathrm{BP}$ was essential in maintaining tissue perfusion in the face of increased systemic resistance. The term "essential hypertension" ${ }^{\prime \prime}$ thus came into being. The homeostatic mechanisms in the human body tend to maintain the blood flow rather than the BP. We can only have an optimal blood flow and not optimal BP. 
But BP is also important because a high pressure can induce shear strain on the vessel wall, which can produce atherosclerosis. In the pulmonary circulation, the blood flow is the same as systemic circulation, but pressure is much lower, and atherosclerosis is almost unknown. Also, reduction of $\mathrm{BP}$ in hypertensive individuals has been shown to have beneficial effects on cardiovascular (CV) and renal outcomes. So, an ideal circulation ensures adequate flow to the tissues at the lowest pressure. ${ }^{6}$ Thus, we can have a J-curve for blood flow, but not for BP. J-curve phenomenon occurs during treatment of hypertension in those patients in whom reduction in BP has resulted in reduction in perfusion. Obviously, it will vary from individual to individual, organ to organ and even from time to time (Fig. 1).

\section{Does a J-Curve Exist?}

There should be a BP below which event rate increases because as the BP reaches zero, life cannot be sustained. The question to be answered is whether this point exists in the BP range that we normally achieve while treating hypertensive patients. Moreover, the fear of the J-curve can lead to undertreatment of hypertension. Across the world, undertreatment of hypertension is a much bigger problem than overtreatment.

In 1979, Stewart published data in Lancet suggesting five times increased risk of myocardial infarction (MI) in hypertensive patients treated to a DBP of less than $90 \mathrm{~mm} \mathrm{Hg}$ compared with those with DBP of 100 to $110 \mathrm{~mm} \mathrm{Hg}$. This was one of the first studies addressing J-curve in hypertension. ${ }^{7}$ In 1987, Cruickshank et al published similar data. ${ }^{8}$ This report suggested an increased risk of $\mathrm{MI}$ in patients with high CV risk when

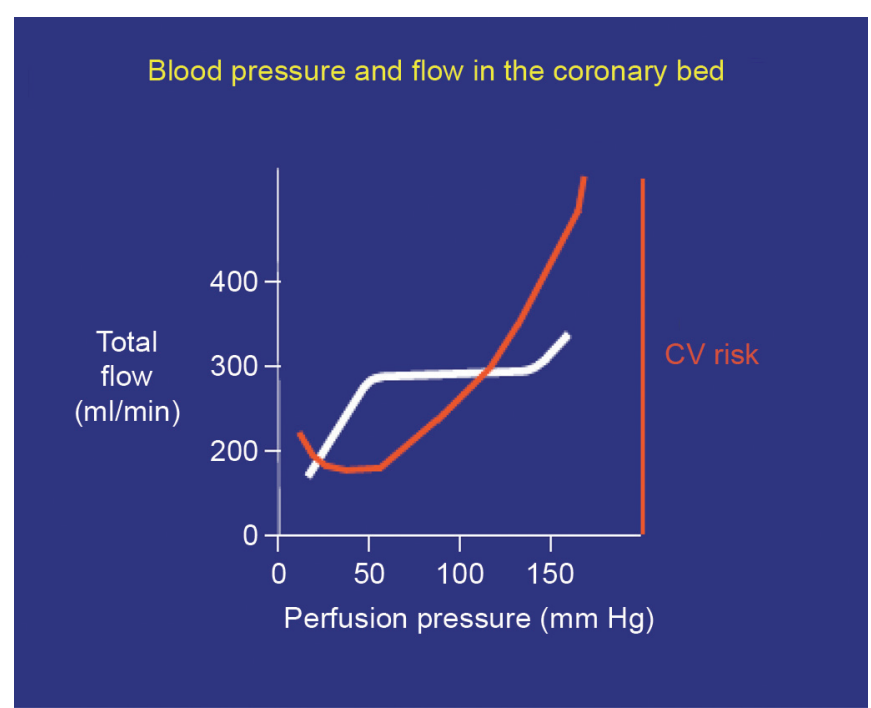

Fig. 1: Coronary flow plotted against perfusion pressure and CV risk. The flow is maintained uniform between a MAP of 50 and $150 \mathrm{~mm}$ $\mathrm{Hg}$. Below $50 \mathrm{~mm} \mathrm{Hg}$, flow reduces. CV risk is the minimum at this point, where there is adequate flow at the lowest perfusion pressure ${ }^{6}$
DBP was reduced below $85 \mathrm{~mm} \mathrm{Hg}$. But there was no such relationship for stroke or renal function. Since then, a large number of trials have been published with conflicting results regarding J-curve for hypertension.

Vital organs such as the heart, kidney, and brain have an autoregulatory capacity to maintain perfusion at a wide range of $\mathrm{BP}$ levels. However, this requires a healthy vessel and healthy endothelium. In the presence of atherosclerosis, the vessels lose this capacity. This may explain the presence of J-curve in patients already having established atherosclerosis and the absence of J-curve in healthy people down to very low levels of BP.

\section{Coronary Circulation and DBP}

As the coronaries are supplied during diastole, heart is likely to have a J-curve for DBP, especially when there is significant obstructive CAD. The coronary perfusion pressure is the difference between coronary artery pressure and left ventricular (LV) diastolic pressure. In patients with obstructive CAD, the driving force will be the pressure distal to the obstruction. Thus, the aortic diastolic pressure may not be a good indicator of the coronary perfusion pressure distal to the obstruction. When there is diastolic dysfunction and the LV diastolic pressure is high, the coronary perfusion pressure falls further. Patients with LV hypertension (LVH) usually have some amount of LV diastolic dysfunction and so the LV end-diastolic pressure is high. Moreover, in LVH, the myocardial capillary density is less and thus relative ischemia exists even otherwise.

In the elderly with ISH, the increased SBP increases the myocardial oxygen demand, while the low DBP reduces the blood supply. However, wide PP is a marker of arterial stiffness, and so, the increased event rate may be caused by the increased aortic stiffness or loss of aortic elasticity rather than the reduced DBP. Thus, the low DBP that predates the treatment may be the cause of the increased event rates rather than the treatment-induced low DBP. It may, however, be prudent not to reduce DBP to low levels in patients with obstructive coronary artery disease, $\mathrm{LVH}$, elderly and those with wide PP (Figs 2A and B).

Let us review some important studies looking at this problem.

\section{Hypertension Optimal Treatment Trial}

Subjects were randomly assigned to three groups of DBP, namely $<90,<85$, and $<80 \mathrm{~mm} \mathrm{Hg}$. A mean BP level of $139 / 82.6 \mathrm{~mm} \mathrm{Hg}$ had the lowest rate of CV events. The lowest incidence of $\mathrm{CV}$ death was at a mean DBP of $86.5 \mathrm{~mm} \mathrm{Hg}$. Reducing DBP to below $82.6 \mathrm{~mm} \mathrm{Hg}$ did not entail any further benefit, but there were no safety issues. Subgroup analysis of 1500 diabetic patients showed that 

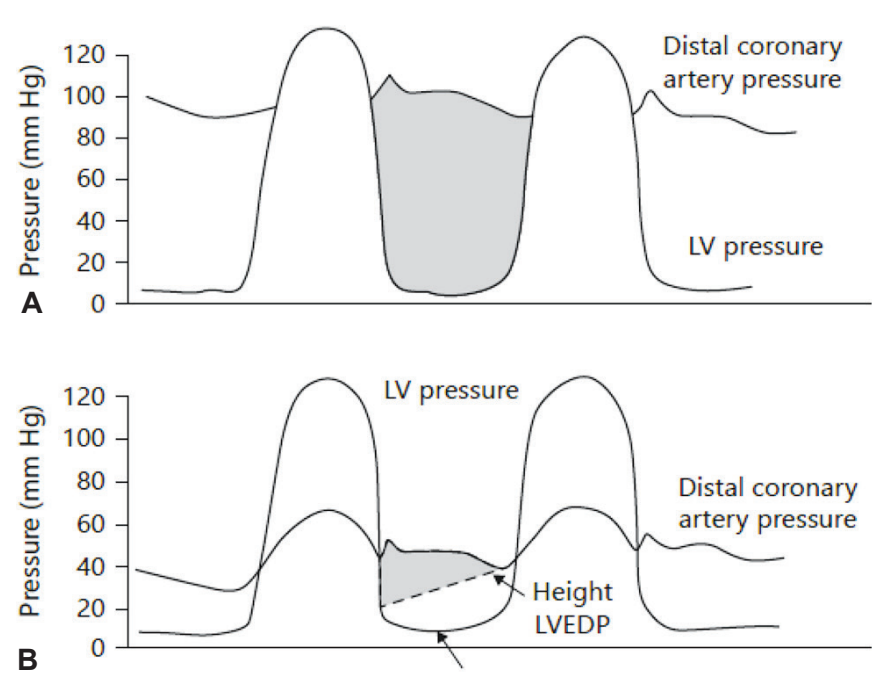

Figs 2A and B: Left ventricular and coronary artery pressures The shaded portion is the coronary perfusion pressure: $(A)$ Normal person and (B) Patient with obstructive CAD and high LV diastolic pressure (Source: Boudoulas H, Leier CV. Clinical perspectives: myocardial perfusion pressure in the age of afterload reduction. ACC Curr J Rev 2000;9(5):27-31)

the rate of major $\mathrm{CV}$ events in those with a DBP of $<80 \mathrm{~mm}$ $\mathrm{Hg}$ was only half of those with $\mathrm{DBP}<90 \mathrm{~mm} \mathrm{Hg}$. A post hoc analysis published in 1998 showed that in the subgroup of patients with ischemic heart disease, there was an increase in MI frequency below a DBP of $83 \mathrm{~mm} \mathrm{Hg} .{ }^{9}$

\section{VALUE Trial}

Hypertensive patients at a high CV risk had higher CV event rates when SBP was reduced below $120 \mathrm{~mm} \mathrm{Hg}{ }^{10}$ However, further analysis of the data published recently ${ }^{11}$ showed that after covariate adjustment, although DBP more than $90 \mathrm{~mm} \mathrm{Hg}$ compared with less than $90 \mathrm{~mm} \mathrm{Hg}$ was associated with an increased incidence of primary $\mathrm{CV}$ end points, DBP less than $70 \mathrm{~mm} \mathrm{Hg}$ compared with more than $70 \mathrm{~mm} \mathrm{Hg}$ was not associated with increased incidence. Similar results were shown with death, MI stroke, and heart failure analyzed separately. Nadir DBP for MI was $76 \mathrm{~mm} \mathrm{Hg}$ and for stroke $60 \mathrm{~mm} \mathrm{Hg}$. Ratio of MI to stroke increased with lower DBP and this was more pronounced in patients with CAD. No J-curve could be demonstrated in either group.

\section{INVEST}

This large study included 22,576 hypertensive patients with stable CAD. The primary outcomes (death, MI, stroke) decreased when DBP was decreased to 80 to $89 \mathrm{~mm}$ $\mathrm{Hg}$. As BP levels reached even lower, CV events increased. Diastolic J-curve was more pronounced than the systolic J-curve. This was very evident when DBP reached less than $60 \mathrm{~mm} \mathrm{Hg}$. As the SBP was progressively reduced, stroke rate came down till $120 \mathrm{~mm} \mathrm{Hg}$. But, below 130 $\mathrm{mm} \mathrm{Hg}, \mathrm{MI}$ risk increased, suggesting a J-curve for MI. The nadir for SBP was $119 \mathrm{~mm} \mathrm{Hg}$. The J-curve was less evident in revascularized patients. ${ }^{12}$

\section{ON TARGET}

The trial studied 25,620 hypertensive patients with previous $\mathrm{CV}$ events or diabetic patients with organ damage. As BP came down from 145/82 to 133/76 mm $\mathrm{Hg}$, the CV events came down. Further reduction in BP increased $\mathrm{CV}$ events, except stroke. ${ }^{13}$

\section{ACCORD}

The study was conducted in 4733 patients with type 2 diabetes mellitus (DM). There was no benefit when SBP was reduced from 133 to $119 \mathrm{~mm} \mathrm{Hg}$. However, there was no increase in event rate. J-curve was not apparent. Stroke was reduced in the aggressive treatment arm. ${ }^{14}$

\section{SPRINT}

This is the latest in the series of hypertension trials looking at the existence of J curve. Probably going to be a game changer, this huge NIH-sponsored trial had to be prematurely stopped because of significant benefit in the arm that reduced mean SBP to $121.4 \mathrm{~mm} \mathrm{Hg}$. Patients with DM, prior stroke and polycystic kidney were excluded. The standard group achieved an SBP of less than $140 \mathrm{~mm} \mathrm{Hg}$, while the intensive treatment group had an SBP target of less than $120 \mathrm{~mm} \mathrm{Hg}$, but attained a mean SBP of $121.4 \mathrm{~mm} \mathrm{Hg}$. The reduction in cardiac events was almost one-third and all-cause mortality was reduced by one-fourth in the group wherein mean SBP was $121.4 \mathrm{~mm} \mathrm{Hg}^{15}$

\section{Lancet Meta-analysis}

This meta-analysis included 123 studies with 613,815 participants. ${ }^{16}$ Every $10 \mathrm{~mm} \mathrm{Hg}$ reduction in SBP resulted in a significant reduction in risk of major CVD events, coronary heart disease, stroke, and heart failure. There was a $13 \%$ reduction in overall mortality. The risk reduction was across various baseline BP levels and comorbidities. The results support reducing SBP to below $130 \mathrm{~mm} \mathrm{Hg}$. The benefit was seen in patients with a history of CVD, coronary heart disease, stroke, diabetes, heart failure, and chronic kidney disease.

\section{J-Curve for Stroke}

Despite all these data suggesting a J-curve for CV end points, the data for a J-curve for stroke are less convincing. There is a strong linear relationship with both SBP and DBP and stroke across a wide range of 
$\mathrm{BP}^{14}$ In the PROGRESS study, lowest risk of stroke was in those in the lowest quarter of BP $(112 / 72 \mathrm{~mm} \mathrm{Hg}) .{ }^{17}$ The autoregulation of brain is probably better than heart. The cerebral vessels are able to maintain perfusion at lower $\mathrm{BP}$ levels than coronary vessels. However, in a posthoc analysis of PROFESS trial, patients with a very low SBP of less than $120 \mathrm{~mm} \mathrm{Hg}$ had more CV events and stroke than those with an SBP of 130 to $139 \mathrm{~mm} \mathrm{Hg} .{ }^{18}$ Thus, for a final word on J curve for stroke, more data are required.

\section{J-Curve and Renal Perfusion}

It is well recorded that systolic hypertension accelerates progression of kidney disease. Most of the trials focused on progression of albuminuria or decline in glomerular filtration rate (GFR). Randomized controlled data for identifying the lowest pressure at which the progression of kidney disease is reduced to the maximum are absent. As renal perfusion occurs in systole, it is unlikely that there will be a diastolic J-curve for renal events. But abrupt lowering of BP, especially in patients with CKD, can produce sudden fall in glomerular filtration pressure and thus GFR. It may be noted that in the SPRINT trial, acute kidney injury or failure was more in the intensive treatment arm. ${ }^{15}$ This trial has created a rethinking on J-curve for renal disease.

\section{Isolated Systolic Hypertension}

The importance of J-curve for DBP is even more in this subgroup that already has a low DBP. By treatment of SBP, if DBP falls to low levels, CV event rates might increase. Current guidelines recommend that we should try to bring down the SBP. Usually, the fall in SBP will be much greater than the fall in DBP. Wang et $\mathrm{al}^{19}$ showed that antihypertensive treatment reduced stroke, MI and other CV events in all ages and across all BP levels. The absolute benefit actually increased in the elderly, who had a lower DBP. Patients with significant reduction in SBP showed consistent reduction of all outcomes, which was independent of the fall in DBP or achieved DBP. This was true, even when the DBP was less than $70 \mathrm{~mm} \mathrm{Hg}$. Kannel et al, ${ }^{20}$ using Framingham data, identified that J-curve for DBP below $80 \mathrm{~mm} \mathrm{Hg}$ existed only for those whose SBP was more than 140 or $160 \mathrm{~mm} \mathrm{Hg}$. Thus, the authors feel that the J curve is related to the wide PP rather than the low DBP. Finally, it is worth remembering that ISH patients have BP, which is most difficult to control, but gets the highest absolute benefit from BP reduction.

\section{J-Curve and CAD}

Most of the data suggest a J-curve in this group. As already explained, in patients with significant obstructive CAD, it is likely that a J-curve exists. But as the SBP is simultaneously brought down, the myocardial oxygen demand is also reduced. J-curve is less apparent in revascularized patients. ${ }^{21}$ The explanations of increased aortic stiffness and reverse causality are also applicable in this group. Careful titration of drugs, keenly watching any worsening of angina and revascularization if indicated, may be the right answer. As a bonus, such careful treatment may reduce stroke.

The data which show that lower DBP led to an increased $\mathrm{CV}$ event rate do not necessarily mean that therapeutic lowering of DBP was the cause of increased event rate. Patients with a low DBP are likely to be older, have diabetes, more likely to have coronary artery disease, high SBP, and wide PP. ${ }^{22}$ These comorbid conditions may be responsible for the increased event rate. Whether overtreatment of hypertension is the cause of J curve phenomenon or the comorbid conditions are the cause of low BP as well as the increased event rate (reverse causality) is yet to be clearly answered.

\section{CONCLUSION}

Physiologically, J-curve should exist because there has to be a BP below which it is incompatible with life. Does this occur in the range of $\mathrm{BP}$ that we attain during treatment is the question. Although the various trials do not give a definite answer, we can arrive at some conclusions. Evidence for a J-curve for stroke is sparse (notable exception being the PROFESS study). For renal end points, questions about J-curve have come to the forefront again after publication of SPRINT results. J-curve for SBP appears unlikely, though as alluded to earlier, there are some data for J curve for SBP also. There appears to be a J-curve for DBP for patients with significant obstructive CAD. This becomes less apparent on revascularization. In patients with non-revascularized, obstructive CAD and in patients with ISH and LVH, care should be taken when DBP falls below $70 \mathrm{~mm} \mathrm{Hg}$ and definitely below $60 \mathrm{~mm} \mathrm{Hg}$.

\section{REFERENCES}

1. White P. Heart disease. 1st ed. New York, NY: McMillan; 1931.

2. Tice F. Practice of medicine. 1st ed. Hagerstown, MD: WF Prior Company, Inc.; 1924.

3. Franklin SS. Isolated systolic hypertension and the J-curve of cardiovascular disease risk. Artery Res 2010 Mar;4(1):1-6.

4. Franklin SS, Lopez VA, Wong ND, Mitchell GF, Larson MG, Vasan RS, Levy D. Single versus combined blood pressure components and risk for cardiovascular disease: the Framingham heart study. Circulation 2009 Jan 20;119(2):243-250.

5. Pickering G. High blood pressure. 2nd ed. London: Churchill; 1968.

6. Giles TD. It is all about flow or, why there will never be one optimal blood pressure goal for every patient with hypertension. J Clin Hypertens (Greenwich) 2011 Jun;13(6): 395-396. 
7. Stewart IM. Relation of reduction in pressure to first myocardial infarction in patients receiving treatment for severe hypertension. Lancet 1979 Apr 21;1(8121):861-865.

8. Cruickshank JM, Thorp JM, Zacharias FJ. Benefits and potential harm of lowering blood pressure. Lancet $1987 \mathrm{Mar}$ 14;1(8533):581-584.

9. Hansson L, Zanchetti A, Carruthers SG, Dahlof B, Elmfeldt D, Julius S, Menard J, Rahn KH, Wedel H, Westerling S. Effects of intensive blood-pressure lowering and low-dose aspirin in patients with hypertension: principal results of the Hypertension Optimal Treatment (HOT) randomised trial. HOT Study Group. Lancet 1998 Jun 13;351(9118): 1755-1762.

10. Julius S, Kjeldsen SE, Weber M, Brunner HR, Ekman S, Hansson L, Hua T, Laragh J, Mclnnes GT, Mitchell L, et al. Outcomes in hypertensive patients at high cardiovascular risk treated with regimens based on valsartan or amlodipine: the VALUE randomized trial. Lancet2004Jun 19;363(9426): 2022-2031.

11. Kjeldsen SE, Berge E, Bangalore S, Messerli FH, Mancia G, Holzhauer B, Hua TA, Zappe D, Zanchetti A, Weber MA, et al. No evidence for a J-shaped curve in treated hypertensive patients with increased cardiovascular risk: the VALUE trial. Blood Press. 2015 Oct 29:1-10.

12. Pepine CJ, Handberg EM, Cooper-DeHoff RM, Marks RG, Kowey P, Messerli FH, Mancia G, Cangiano JL, Garcia-Barreto $\mathrm{D}$, Keltai M, et al. A calcium antagonist versus a non-calcium antagonist hypertension treatment strategy for patients with coronary artery disease. The International VerapamilTrandolapril Study (INVEST): a randomized controlled trial. JAMA 2003;290:2805-2816.

13. Sleight $\mathrm{P}$, Redon J, Verdecchia $\mathrm{P}$, et al. Prognostic value of blood pressure in patients with high vascular risk in the Ongoing Telmisartan Alone and in combination with Ramipril Global Endpoint Trial study. J Hypertens 2009 Jul;27(7):1360-1369.
14. Cushman WC, Evans GW, Byington RP, Goff DC Jr, Grimm RH Jr, Cutler JA, Simons-Morton DG, Basile JN, Corson MA, Probstfield JL, et al. The ACCORD study group: effects of intensive blood-pressure control in type 2 diabetes mellitus. N Engl J Med 2010 Apr 29;362(17):1575-1585.

15. The SPRINT Research Group. A randomized trial of intensive versus standard blood-pressure control. N Engl J Med 2015 Nov 26;373(22):2103-2116.

16. Ettehad D, Emdin CA, Kiran A, Anderson SG, Callender T, Emberson, Chalmers J, Rodgers A, Rahimi K. Blood pressure lowering for prevention of cardiovascular disease and death: a systematic review and meta-analysis. Lancet 2015 Dec 23.

17. Arima $\mathrm{H}$, Chalmers J, Woodward $\mathrm{M}$, Anderson $\mathrm{C}$, Rodgers A, Davis S, Macmahon S, Neal B. PROGRESS Collaborative Group. Lower target blood pressures are safe and effective for the prevention of recurrent stroke: the PROGRESS trial. J Hypertens 2006 Jun;24(6):1201-1208.

18. Ovbiagele B, Diener HC, Yusuf S, Martin RH, Cotton D, Vinisko R, Donnan GA, Bath PM. PROFESS Investigators. Level of systolic blood pressure within the normal range and risk of recurrent stroke. JAMA 2011 Nov 16;306(19):2137-2144.

19. Wang J-G, Staessen JA, Franklin SS, Fagard R, Gueyffier F. Systolic and diastolic blood pressure lowering as determinants of cardiovascular outcome. Hypertension 2005 May;45(5): 907-913.

20. Kannel WB, Wilson PW, Nam BH, D'Agostino RB, Li J. A likely explanation for J curve of blood pressure cardiovascular risk. Am J Cardiol 2004 Aug;94(3):3804.

21. Messerli FH, Mancia G, Conti CR, Hewkin AC, Kupfer S, Champion A, Kolloch R, Benetos A, Pepine CJ. Dogma disputed: can aggressively lowering blood pressure for hypertension in hypertensive patients with coronary artery disease be dangerous? Ann Intern Med 2006 Jun 20;144(12):884-893

22. Williams B. Hypertension and the J-Curve. J Am Coll Cardiol 2009;54(20):1835-1836. 